\title{
Directional, horizontal inhomogeneities of cloud optical thickness fields retrieved from ground-based and airbornespectral imaging
}

\author{
Michael Schäfer $^{1}$, Eike Bierwirth ${ }^{1, a}$, André Ehrlich ${ }^{1}$, Evelyn Jäkel ${ }^{1}$, Frank Werner ${ }^{2}$, and Manfred Wendisch ${ }^{1}$ \\ ${ }^{1}$ Leipzig Institute for Meteorology, University of Leipzig, Leipzig, Germany \\ ${ }^{2}$ Joint Center for Earth Systems Technology, University of Maryland, 5523 Research Park Drive 320, \\ Baltimore, MD 21228, USA \\ anow at: PIER-ELECTRONIC GmbH, Nassaustr. 33-35, 65719 Hofheim-Wallau, Germany
}

Correspondence to: Michael Schäfer (michael.schaefer@uni-leipzig.de)

Received: 16 August 2016 - Discussion started: 26 August 2016

Revised: 15 December 2016 - Accepted: 28 December 2016 - Published: 15 February 2017

\begin{abstract}
Clouds exhibit distinct horizontal inhomogeneities of their optical and microphysical properties, which complicate their realistic representation in weather and climate models. In order to investigate the horizontal structure of cloud inhomogeneities, 2-D horizontal fields of optical thickness $(\tau)$ of subtropical cirrus and Arctic stratus are investigated with a spatial resolution of less than $10 \mathrm{~m}$. The 2$\mathrm{D} \tau$-fields are derived from (a) downward (transmitted) solar spectral radiance measurements from the ground beneath four subtropical cirrus and (b) upward (reflected) radiances measured from aircraft above 10 Arctic stratus. The data were collected during two field campaigns: (a) Clouds, Aerosol, Radiation, and tuRbulence in the trade wind regime over BArbados (CARRIBA) and (b) VERtical Distribution of Ice in Arctic clouds (VERDI). One-dimensional and 2-D autocorrelation functions, as well as power spectral densities, are derived from the retrieved $\tau$-fields. The typical spatial scale of cloud inhomogeneities is quantified for each cloud case. Similarly, the scales at which 3-D radiative effects influence the radiance field are identified. In most of the investigated cloud cases considerable cloud inhomogeneities with a prevailing directional structure are found. In these cases, the cloud inhomogeneities favour a specific horizontal direction, while across this direction the cloud is of homogeneous character. The investigations reveal that it is not sufficient to quantify horizontal cloud inhomogeneities using 1-D inhomogeneity parameters; 2-D parameters are necessary.
\end{abstract}

\section{Introduction}

The globally and annually averaged cloud cover is in the range of about $70 \%$ (Rossow and Schiffer, 1999). Because of this high percentage, and the important effect of cloud cover on Earth's radiation budget, clouds need to be considered as an important regulator of Earth's climate (Albrecht, 1989; Loeb et al., 2009). Clouds scatter and absorb solar radiation in the wavelength range from 0.2 to $5 \mu \mathrm{m}$; they emit and absorb terrestrial radiation from 5 to $50 \mu \mathrm{m}$. Although clouds have been studied for several decades, they are still poorly represented in weather and climate models (Shonk et al., 2011). The latest report of the Intergovernmental Panel on Climate Change (IPCC) classifies cloud effects as one of the largest uncertainties in climate simulations, significantly contributing to problems in the determination of Earth's energy budget (Stocker et al., 2013). These issues partly arise from an unrealistic representation of complex horizontal cloud structures and from cloud-radiation feedback processes that control the cloud evolution (Stephens, 2005; Shonk et al., 2011). Therefore, the representation of cloud inhomogeneities needs to be more realistic (Shonk et al., 2011). This is particularly important because changes of cloud properties may have serious consequences for the interaction of clouds with radiation (Slingo, 1990).

Several independent studies investigated the influence of the plane-parallel assumption on cloud retrievals (e.g. Cahalan, 1994; Loeb and Davies, 1996; Marshak et al., 1998; Zinner et al., 2006; Varnai and Marshak, 2007). They found that 
the magnitudes of model biases are related to the degree of horizontal photon transport. In 1-D radiative transfer simulations, clouds are divided into separate vertical columns with horizontal homogeneous optical and microphysical properties (independent pixel approximation, IPA). However, horizontal photon transport cannot be neglected in case of inhomogeneous clouds. Additionally, multiple cases of scattering due to 3-D microphysical cloud structures smooth the horizontal radiation field. On small scales, this limits the accuracy of IPA. For example, Cahalan (1994) and Marshak et al. (1995) revealed discrepancies for individual pixel radiances exceeding $50 \%$ due to a plan-parallel bias.

High ice clouds (cirrus) and Arctic stratus exhibit horizontal inhomogeneities at different horizontal scales. Both cloud types can either warm or cool Earth's climate system, depending on their optical and microphysical properties and the meteorological conditions. For example, Choi and Ho (2006) reported for tropical regions a positive (warming) net radiative effect of cirrus for a cirrus optical thickness $\left(\tau_{\mathrm{ci}}\right)$ of less than 10, but a cooling effect for $\tau_{\mathrm{ci}}>10$. For Arctic stratus, Wendisch et al. (2013) showed that for low surface albedo $\left(\alpha_{\mathrm{s}}\right)$ and low solar zenith angle $\left(\theta_{0}\right)$, the cloud cools the subcloud layer. With increasing $\alpha_{\mathrm{s}}$ and increasing $\theta_{0}$, the cooling effect of the low-level cloud turns into a warming. Both clouds and surface reflection properties can vary significantly on different horizontal scales.

In many remote-sensing applications clouds are assumed to be plane-parallel (Francis et al., 1998; Iwabuchi and Hayasaka, 2002; Garrett et al., 2003), which may introduce biases into the modelled radiation budget (Shonk et al., 2011). For example, in the case of cirrus, Carlin et al. (2002) found a plane-parallel cirrus albedo bias of up to $25 \%$ due to spatial cirrus inhomogeneity. For Arctic stratus over variable sea-ice surfaces, Rozwadowska and Cahalan (2002) reported a plane-parallel albedo bias of less than $2 \%$, but an absolute value of the transmittance bias that could exceed $10 \%$.

Three-dimensional Monte Carlo radiative transfer simulations account for horizontal photon transport (Barlakas et al., 2016). However, they are costly in terms of computational time and memory (Huang and Liu, 2014). This renders Monte Carlo radiative transfer simulations inappropriate for application in operational or global models. Other approaches introduce Monte Carlo integration of independent column approximation (McICA), as proposed by Pincus et al. (2003). McICA is a computational efficient technique for computing domain-averaged broadband radiative flux densities in vertically and horizontally variable cloud fields (Pincus et al., 2003). Improvements compared to the plane-parallel assumption are achieved with this approach, but results are still not as accurate as those from 3-D Monte Carlo models. To reduce uncertainties associated with the 1D plane-parallel assumption, Huang and Liu (2014) applied spatial autocorrelation functions of cloud extinction coefficients to capture the net effects of sub-grid cloud interactions with radiation. With several orders less of computation time, this approach reproduces 3-D Monte Carlo radiative transfer simulations with an accuracy within $1 \%$. However, Huang and Liu (2014) assumed perfect knowledge about the spatial correlation functions of cloud extinction coefficients, which underlines the need for measurements of comparable resolved inhomogeneity measures.

General circulation or numerical weather forecast models require sub-grid scale parameterization of cloud structures, liquid water content (LWC), and/or ice water content (IWC), for example (Huang and Liu, 2014). In reality, cloud structures reveal spatial features down to distances below the metre scale (Pinsky and Khain, 2003). Therefore, measurements with appropriate spatial and temporal resolution have to be conducted in order to derive the needed parameterizations. The required measurements include cloud altitude (temperature), its geometry (vertical and horizontal extent), and cloud microphysical properties (e.g., LWC, IWC, droplet size, ice crystal size, and shape distributions).

Cloud inhomogeneities often exhibit a typical directional structure (e.g., induced by the prevailing wind). In such a case, 1-D observations with lidar (light detecting and ranging) or point spectrometers can lead to an underestimation or overestimation of the degree of cloud inhomogeneity of the whole cloud scene. For example, a cloud with a rather inhomogeneous character may be classified as horizontally homogeneous (underestimation of inhomogeneity) if the dominating cloud structure has the same orientation as the cloud observational path. Conversely, the cloud inhomogeneity would be overestimated if the cloud were scanned perpendicular to the major directional structure. Therefore, 2-D observations are a useful tool for avoiding such misinterpretations of cloud inhomogeneity.

In this paper, horizontal $\tau$ fields retrieved from solar spectral radiance measurements are analysed to quantify horizontal inhomogeneities of two cloud types: subtropical cirrus and Arctic stratus. The information content of 1-D and 2-D approaches to cloud inhomogeneity analysis is compared to identify their scientific value and limits. In Sect. 3, a statistical evaluation of the horizontal inhomogeneity of the fields of $\tau$ is presented using common 1-D inhomogeneity parameters from the literature. Those bulk properties are valid to quantify the overall cloud inhomogeneity but cannot reproduce spatial inhomogeneities of the cloud field. In Sect. 4, the derived bulk properties from the 1-D inhomogeneity parameters are compared to 1-D and 2-D autocorrelation functions. Finally, in Sect. 5, 1-D and 2-D Fourier analysis is used to investigate the effect of horizontal cloud inhomogeneities on radiative transfer.

\section{Data set: 2-D fields of cloud optical thickness}

Data from two international field campaigns were analysed: the Clouds, Aerosol, Radiation, and Turbulence in the Trade Wind Regime over Barbados (CARRIBA, Siebert et al., 
2013; Schäfer et al., 2013) campaign performed on Barbados in April 2011 and the Vertical Distribution of Ice in Arctic Clouds (VERDI, Schäfer et al., 2015) observations carried out in Inuvik, Canada, in May 2012. Two-dimensional fields of downward and upward solar spectral radiances $\left(I_{\lambda}^{\downarrow}\right.$, $I_{\lambda}^{\uparrow}$ ) were measured from the ground (CARRIBA) and from an aircraft (VERDI). The imaging spectrometer AisaEAGLE (manufactured by Specim Ltd., Finland; Hanus et al., 2008; Schäfer et al., 2013, 2015) was used for the measurements. It is a single-line sensor with a field of view (FOV) of $37^{\circ}$ and 1024 spatial pixels detecting radiation in the wavelength range from 400 to $970 \mathrm{~nm}$ with a spectral resolution of $1.25 \mathrm{~nm}$ full width at half maximum (FWHM). The 2-D scans of the cloud scenes are generated from sequential (4 to $30 \mathrm{~Hz}$ frame rate) measurements of the single sensor-line, while the target (cloud) moves with the wind (ground-based) or the flying aircraft across this sensor line. Adding up all measured lines behind each other, the 2-D scan evolves as an image with a spatial (number of sensor pixels) and temporal (number of recorded frames) axis. Applying the known geometry, integration time, and cloud and aircraft velocities, the axis dimensions can be transferred into distances. The 2-D images evolved either from the heading of the clouds above the sensor line (ground-based) or by the movement of the sensor line itself above the clouds (airborne). The imaging spectrometer was characterized and calibrated in the laboratory to transform the AisaEAGLE's raw data (12-bit digital numbers) into radiance. The procedure of data evaluation (calibrations, corrections) follows the methods described by Bierwirth et al. (2013) and Schäfer et al. (2013, 2015).

As proposed by Marshak et al. (1995), Oreopoulos et al. (2000), or Schröder (2004), horizontal cloud inhomogeneities are studied by scale analysis of cloud-top reflectances. However, radiance measurements include the information of the scattering phase function (e.g., forwardbackward scattering peak, halo features) in the measured fields of radiance (Schäfer et al., 2013). To avoid artefacts in the scale analysis resulting from such features, parameters that are independent of the directional scattering of the cloud particles have to be analysed. The cloud optical thickness $\tau$ does not include the fingerprint of the scattering phase function. Therefore, the ground-based and airborne measured fields of $I_{\lambda}^{\downarrow}$ (CARRIBA) and $I_{\lambda}^{\uparrow}$ (VERDI) were used to retrieve horizontal fields of $\tau$ with a spatial resolution of less than $10 \mathrm{~m}$. The retrieved fields of $\tau$ were then applied to investigate horizontal cloud inhomogeneities of subtropical cirrus (index ci) and Arctic stratus (index st).

Simulations were performed with the radiative transfer solver DISORT 2 (Discrete Ordinate Radiative Transfer). Input parameters such as cloud optical properties, aerosol content, and spectral surface albedo are provided by the library for radiative transfer calculations (libRadtran, Mayer and Kylling, 2005). The required profiles of thermodynamic parameters are derived from measurements from radiosondes and/or dropsondes. Despite assuming plane-parallel clouds in the simulations, the investigation of 3-D radiative effects is still possible using the retrieved fields of $\tau$, but directional features related to the scattering phase function are avoided. $I_{\lambda}^{\downarrow}$ and $I_{\lambda}^{\uparrow}$ were simulated as a function of values of $\tau_{\mathrm{ci}}$ and $\tau_{\text {st }}$, respectively. The simulations were performed for all scattering angles within the FOV of AisaEAGLE. Thus, simulated grids of possible $I_{\lambda}^{\downarrow}$ and $I_{\lambda}^{\uparrow}$ and corresponding $\tau_{\mathrm{ci}}$ and $\tau_{\mathrm{st}}$ are available for each time step of the measurements and each spatial pixel. The retrieved $\tau_{\mathrm{ci}}$ and $\tau_{\mathrm{st}}$ are derived by interpolating the simulated $I_{\lambda}^{\downarrow}$ and $I_{\lambda}^{\uparrow}$ to the measured value for each spatial pixel using a linear interpolation. More detailed descriptions and sensitivity tests of the applied retrieval procedures are reported by Schäfer et al. (2013) for subtropical cirrus and by Bierwirth et al. (2013) and Schäfer et al. (2015) for Arctic stratus. Fields of cloud optical thickness are derived for four subtropical cirrus cases $\left(\tau_{\mathrm{ci}}\right)$ and $10 \mathrm{Arc}-$ tic stratus cases $\left(\tau_{\mathrm{st}}\right)$. Subsequently, those fields of $\tau_{\mathrm{ci}}$ and $\tau_{\mathrm{st}}$ are used to investigate and quantify horizontal cloud inhomogeneities.

Table 1 summarizes the statistical parameters of the four retrieved fields of $\tau_{\mathrm{ci}}$ ( $\mathrm{Ci}-01$ to $\left.\mathrm{Ci}-04\right)$ and the 10 retrieved fields of $\tau_{\mathrm{st}}$ (St-01 to St-10). Figure 1 illustrates example cutouts for cases St-04 and St-07, both characterized by a measurement duration of $60 \mathrm{~s}$. Table 1 further provides information on the measurement time, cloud altitude $\left(h_{\mathrm{cld}}\right)$, field size (swath, length), and average and standard deviation of $\tau_{\mathrm{ci}}\left(\bar{\tau}_{\mathrm{ci}} \pm \sigma_{\tau, \mathrm{ci}}\right)$ and $\tau_{\mathrm{st}}\left(\bar{\tau}_{\mathrm{st}} \pm \sigma_{\tau, \mathrm{st}}\right)$. The sampled subtropical cirrus fields of about $13-44 \mathrm{~km}$ length and 7-8 km width are determined by the time of observation and the swath covered by AisaEAGLE. For the Arctic stratus cases the average swath of the covered cloud fields has a size close to $1.3 \mathrm{~km}$. The length varies from 4 to up to $26 \mathrm{~km}$. Thus, for CARRIBA and VERDI sufficiently large areas of the clouds are covered to provide a statistically firm analysis of $\tau_{\mathrm{ci}}$ and $\tau_{\mathrm{st}}$ and to investigate their horizontal inhomogeneities.

\section{One-dimensional inhomogeneity parameters}

The standard deviation $\sigma_{\tau}$ of the cloud optical thickness does not allow a comparison between cases with different average cloud optical thickness $\bar{\tau}$. A cloud with higher $\bar{\tau}$ can exhibit a higher standard deviation. Therefore, similar to the studies by Barker et al. (1996), who used ratios between mean $\tau$ and the variance of $\tau$, Davis et al. (1999a) and Szczap et al. (2000) utilized the normalized inhomogeneity measure $\rho_{\tau}$ to quantify the horizontal inhomogeneity of $\tau$. It is defined by the ratio of $\sigma_{\tau}$ and the average value $\bar{\tau}$ of the corresponding sample:

$\rho_{\tau}=\frac{\sigma_{\tau}}{\bar{\tau}}$.

A homogeneous cloud is characterized by $\rho_{\tau}=0$. Increasing values of $\rho_{\tau}$ indicate rising cloud inhomogeneity. However, 
Table 1. Label, measurement period (day and time of day in UTC), cloud top altitude, pixel size (width, length), domain size (swath, length), and average and standard deviation $\left(\bar{\tau} \pm \sigma_{\tau}\right)$ of the retrieved fields of $\tau_{\mathrm{ci}}$ and $\tau_{\mathrm{st}}$ from ground-based measured CARRIBA (Ci-01 to Ci-04) and airborne measured VERDI (St-01 to St-10) cases. The flight altitude for each VERDI case was at $2920 \mathrm{~m}$. The right three columns include calculated 1-D inhomogeneity parameters $\left(\rho_{\tau}, S_{\tau}, \chi_{\tau}\right)$ of the retrieved fields of $\tau$. They are discussed in Sect. 3.

\begin{tabular}{lllrrrr|rrr}
\hline Case & Day & Time $(\mathrm{UTC})$ & $h_{\text {cld }}(\mathrm{km})$ & Pixel size $(\mathrm{m})$ & Domain $(\mathrm{km})$ & $\bar{\tau} \pm \sigma_{\tau}$ & $\rho_{\tau}$ & $S_{\tau}$ & $\chi_{\tau}$ \\
\hline Ci-01 & 9 April 2011 & $13: 26: 26-13: 37: 13$ & $11-15$ & $\approx 7.1 \times 4.8$ & $7.3 \times 15.6$ & $0.41 \pm 0.17$ & 0.40 & 0.19 & 0.92 \\
Ci-02 & 16 April 2011 & $13: 44: 29-14: 17: 42$ & $12-15$ & $\approx 7.8 \times 5.1$ & $8.0 \times 40.5$ & $0.28 \pm 0.09$ & 0.35 & 0.15 & 0.94 \\
Ci-03 & 18 April 2011 & $13: 43: 56-14: 17: 13$ & $13-15$ & $\approx 8.4 \times 5.5$ & $8.6 \times 44.1$ & $0.20 \pm 0.03$ & 0.17 & 0.08 & 0.99 \\
Ci-04 & 23 April 2011 & $16: 45: 10-17: 03: 12$ & $11-14$ & $\approx 7.1 \times 3.1$ & $7.3 \times 13.3$ & $0.05 \pm 0.04$ & 0.91 & 0.48 & 0.63 \\
\hline St-01 & 14 May 2012 & $20: 31: 47-20: 36: 31$ & $\leq 0.90$ & $\approx 2.7 \times 2.5$ & $1.34 \times 21.28$ & $9.93 \pm 1.89$ & 0.19 & 0.08 & 0.98 \\
St-02 & 14 May 2012 & $20: 38: 04-20: 42: 09$ & $\leq 0.85$ & $\approx 2.8 \times 2.8$ & $1.37 \times 20.83$ & $7.82 \pm 2.01$ & 0.26 & 0.11 & 0.97 \\
St-03 & 14 May 2012 & 20:53:26-20:58:30 & $\leq 0.85$ & $\approx 2.8 \times 3.0$ & $1.37 \times 26.85$ & $3.82 \pm 1.33$ & 0.34 & 0.20 & 0.92 \\
St-04 & 15 May 2012 & $18: 41: 53-18: 43: 58$ & $\leq 1.00$ & $\approx 2.6 \times 2.8$ & $1.27 \times 10.50$ & $14.34 \pm 2.54$ & 0.18 & 0.08 & 0.98 \\
St-05 & 15 May 2012 & 21:05:10-21:09:24 & $\leq 0.93$ & $\approx 2.7 \times 2.7$ & $1.32 \times 20.22$ & $6.35 \pm 0.97$ & 0.15 & 0.07 & 0.99 \\
St-06 & 16 May 2012 & $19: 10: 56-19: 15: 56$ & $\leq 1.00$ & $\approx 2.6 \times 2.6$ & $1.27 \times 23.10$ & $6.52 \pm 1.48$ & 0.23 & 0.11 & 0.97 \\
St-07 & 17 May 2012 & $16: 53: 23-16: 56: 06$ & $\leq 0.25$ & $\approx 3.6 \times 2.6$ & $1.75 \times 12.74$ & $3.04 \pm 0.66$ & 0.22 & 0.11 & 0.97 \\
St-08 & 17 May 2012 & $17: 00: 59-17: 06: 15$ & $\leq 1.00$ & $\approx 2.6 \times 2.7$ & $1.27 \times 25.65$ & $5.48 \pm 1.84$ & 0.34 & 0.15 & 0.95 \\
St-09 & 17 May 2012 & $17: 09: 28-17: 10: 38$ & $\leq 2.25$ & $\approx 1.0 \times 2.6$ & $0.48 \times 5.46$ & $7.07 \pm 1.41$ & 0.20 & 0.09 & 0.98 \\
St-10 & 17 May 2012 & $18: 49: 26-18: 50: 16$ & $\leq 0.23$ & $\approx 3.6 \times 2.7$ & $1.76 \times 4.10$ & $4.15 \pm 0.67$ & 0.16 & 0.08 & 0.99 \\
\hline
\end{tabular}
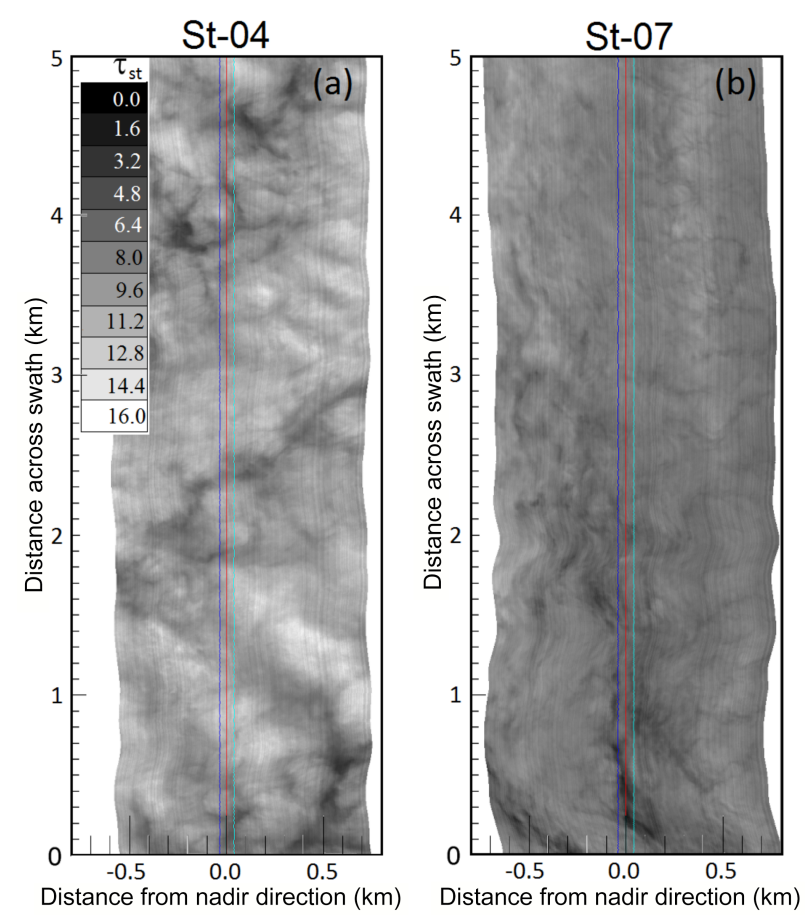

Figure 1. (a) Georeferenced field of $\tau_{\mathrm{st}}$. Cutout from measurement case St-04 with $60 \mathrm{~s}$ measurement duration. The dark blue, red, and light blue lines illustrate the nadir viewing direction in a range of $\pm 1^{\circ}$. (b) Same as panel (a) for case St-07.

$\rho_{\tau}$ has no predefined upper limit, which might lead to misinterpretations in a variability analysis. This renders $\rho_{\tau}$ not as a quantitative but qualitative measure only. Therefore, Davis et al. (1999a) and Szczap et al. (2000) convert the relative variability $\rho_{\tau}$ into the inhomogeneity parameter $S_{\tau}$ as fol- lows:

$$
S_{\tau}=\frac{\sqrt{\ln \left(\rho_{\tau}^{2}+1\right)}}{\ln 10} .
$$

In case of a log-normal frequency distribution of $\tau, S_{\tau}$ is proportional to $\rho_{\tau}$. This is because the reflected or transmitted radiance is approximately linear to $\log \tau$ for moderate $\tau$ (for $\log \tau=0.5-1.5$ with $\tau \approx 3-30$ ). Without net horizontal photon transport, moments of reflected or transmitted radiance are closely linked with moments of $\log \tau$ rather than moments of $\tau$ (Iwabuchi and Hayasaka, 2002). Therefore, $S_{\tau}$ quantifies the degree of cloud inhomogeneity.

Oreopoulos and Cahalan (2005) investigated the inhomogeneity parameter $\chi_{\tau}$, first introduced by Cahalan (1994). $\chi_{\tau}$ is defined as the ratio of the logarithmic and linear average of a distribution of $\bar{\tau}$ :

$\chi_{\tau}=\frac{\exp (\overline{\ln \tau})}{\bar{\tau}}$.

The 1-D inhomogeneity parameter $\chi_{\tau}$ ranges between 0 and 1 , with values close to unity indicating horizontal homogeneity and values approaching zero characterizing high horizontal inhomogeneity. Oreopoulos and Cahalan (2005) state that the reflected solar flux is approximately a linear function of the logarithm of $\tau$ for a wide range of $\tau(\approx 3$ to $\approx 30$, depending on $\theta_{0}$ ). Thus, the logarithmically averaged $\tau$ provides a way to account for cloud inhomogeneity effects in plane-parallel radiative transfer calculations using $\chi_{\tau}$ as a scaling factor with which $\tau$ needs to be multiplied to approximate the IPA albedo.

The three 1-D inhomogeneity parameters $\rho_{\tau}, S_{\tau}$, and $\chi_{\tau}$ are calculated for each retrieved field of $\tau_{\mathrm{ci}}$ and $\tau_{\mathrm{st}}$ from the 
CARRIBA and VERDI campaigns. The results are listed in the right three columns of Table 1 . When comparing them to literature values, one has to keep in mind that cloud inhomogeneities appear on different spatial scales. For example, cloud fields may change on synoptic scales $(\approx 100 \mathrm{~km})$ or dynamic scales (10-100 m) depending on the cloud type. Therefore, inhomogeneity parameters depend on the pixel and domain size of the analysed cloud fields. The larger the domain size or the smaller the pixel size is, the broader the probably density function of the cloud parameter may become. Therefore, a comparison of different cloud cases is only valid when pixel size and cloud domain are in the same range.

The subtropical cirrus cases observed during CARRIBA show $\rho_{\tau}$ in the range of 0.17-0.91, while $S_{\tau}$ is in the range of 0.08-0.48. The largest values of $\rho_{\tau}$ and $S_{\tau}$ are found for Ci04, the lowest for Ci-03. The values for $\rho_{\tau}$ and $S_{\tau}$ show that the subtropical cirrus of $\mathrm{Ci}-02$ and $\mathrm{Ci}-03$ were quite homogeneous, whereas those of $\mathrm{Ci}-01$ and $\mathrm{Ci}-04$ were rather inhomogeneous. For the 10 Arctic stratus cases, $\rho_{\tau}$ and $S_{\tau}$ are in the range of $0.15-0.34$ and $0.07-0.20$, respectively. For stratocumulus days $(6.9 \mathrm{~km}$ domain with $15 \mathrm{~m}$ horizontal resolution), Zuidema and Evans (1998) quantified the inhomogeneity of $\tau$ with $S_{\tau}=0.1-0.3$. Iwabuchi (2000) and Iwabuchi and Hayasaka (2002) investigated the inhomogeneity of $\tau$ for overcast boundary layer clouds using a visible-wavelength moderate-resolution (about $1 \mathrm{~km}$ ) sensor and found values of $S_{\tau}=0.03-0.3$, which lead to $\rho_{\tau}=0.07-0.78$. Thus, considering the different pixel and domain sizes, the derived values from CARRIBA and VERDI compare well with those reported by Zuidema and Evans (1998), Iwabuchi (2000), and Iwabuchi and Hayasaka (2002). Among all 10 cases, $\rho_{\tau}$ and $S_{\tau}$ indicate cases St-03 and St-08 to be more inhomogeneous.

For CARRIBA, the values of $\chi_{\tau}$ range from 0.63 to 0.99 , indicating rather inhomogeneous cirrus for $\mathrm{Ci}-04$ and quite homogeneous cirrus during the other days. In contrast to the results for $\rho_{\tau}, S_{\tau}$ and $\chi_{\tau}$ indicate that the subtropical cirrus of $\mathrm{Ci}-01$ are less inhomogeneous. The calculated values of $\chi_{\tau}$ for the retrieved fields of $\tau_{\mathrm{st}}$ from the VERDI campaign yield values larger than 0.9 in each case, with the lowest values for cases St-03 and St-08, which were already indicated by $\rho_{\tau}$ and $S_{\tau}$ to be more inhomogeneous. Using the Moderate Resolution Imaging Spectroradiometer (MODIS), depending on cloud type, cloud phase, surface type, season, and time of day, Oreopoulos and Cahalan (2005) estimated the range of $\chi_{\tau}$ to be from $\approx 0.65$ to 0.8 at spatial scales of $1^{\circ} \times 1^{\circ}$.

The 1-D inhomogeneity parameters $\rho_{\tau}, S_{\tau}$, and $\chi_{\tau}$ are easy to calculate and suitable for being implemented in simulations that assume horizontally homogeneous clouds to achieve more realistic results. They do not provide a measure of the directional variability of the inhomogeneities. However, different clouds exhibit preferred horizontal inhomogeneity patterns and typical features. For example, the clouds observed during CARRIBA and VERDI are different in terms of cloud altitude, structure, phase, particle size, and shape, although $\rho_{\tau}, S_{\tau}$, and $\chi_{\tau}$ yield comparable values (compare Fig. 1 and Table 1). Therefore, not only the horizontal inhomogeneity but also the spatial coherence of cloud inhomogeneity parameters and their directional dependence need to be investigated (Hill et al., 2012).

\section{Spatial 1-D and 2-D autocorrelation functions and decorrelation length}

The 2-D autocorrelation function $P_{\tau}\left(L_{x}, L_{y}\right)$ is calculated in two horizontal dimensions at fixed distances (pixel lags) $L_{x}$ and $L_{y}$, which are derived as integer multiples of the equidistant sample intervals $x_{j}$ and $y_{k}$ of the 2-D fields of $\tau$ (Marshak et al., 1998). The maximum pixel lags $L_{x}$ and $L_{y}$ are given by the number of pixels $n$ and $m$ of the 2-D fields. Here, with $n$ and $m$ equidistant, measurement intervals $x_{j}$ and $y_{k}, P_{\tau}\left(L_{x}, L_{y}\right)$ for 2-D fields of $\tau$ are calculated by

$$
P_{\tau}\left(L_{x}, L_{y}\right)=\frac{\sum_{j, k+1}^{n, m}\left[\tau\left(x_{j}+L_{x}, y_{k}+L_{y}\right)-\bar{\tau}\right] \times\left[\tau\left(x_{j}, y_{k}\right)-\bar{\tau}\right]}{\sum_{j, k+1}^{n, m}\left[\tau\left(x_{j}, y_{k}\right)-\bar{\tau}\right]^{2}} .
$$

Here, $\tau\left(x_{j}, y_{k}\right)$ is the cloud optical thickness observed at the reference position, and $\tau\left(x_{j}+L_{x}, y_{k}+L_{y}\right)$ is the cloud optical thickness at pixel lags $L_{x}$ and $L_{y}$. The autocorrelation function $P_{\tau}\left(L_{x}, L_{y}\right)$ yields values between -1 and 1 , with 1 representing a perfect positive correlation (e.g., for a spatial shift equal to zero); a value of -1 is a perfect negative correlation and 0 indicates no correlation. Thus, spatial autocorrelation functions quantify the degree of similarity between spatially distributed neighbouring samples. Usually, $\tau$ values in close horizontal distance reveal similar values, while cloud pixels at larger distances may show significantly different values of $\tau$, depending on the cloud heterogeneity. Here, only the degree of correlation matters; the positive or negative sign of the autocorrelation result is of less importance. To avoid misinterpretations with the sign, the squared autocorrelation function $P_{\tau}^{2}\left(L_{x}, L_{y}\right)$ is used here.

Figure $2 \mathrm{~b}$ and $\mathrm{d}$ show examples of $P_{\tau}^{2}\left(L_{x}, L_{y}\right)$ in a 2-D plot for $L_{x}=-250$ to $L_{x}=250$ and $L_{y}=-250$ to $L_{y}=250$, calculated for a selected area (500 by 500 pixels, Fig. $2 \mathrm{a}$ and b) of the cirrus fields from cases $\mathrm{Ci}-01$ and $\mathrm{Ci}-03$ with $L_{x}=250$ and $L_{y}=250$. The positive and negative signs of $L_{x}$ and $L_{y}$ in Fig. 2b and dillustrate the direction into which the particular image is shifted against itself to derive the depicted autocorrelation coefficients. Both cases show a different pattern of $P_{\tau}^{2}\left(L_{x}, L_{y}\right)$ with increasing absolute values of $L_{x}$ and $L_{y}$. While Ci-01 shows a circular spot indicating a symmetry independent of direction, $\mathrm{Ci}-03$ displays high correlation factors for all considered $L_{y}$ values within a range from $L_{x}=-50$ to $L_{x}=50$. This pattern indicates a homogeneous cloud structure along the $y$ axis, while the $\tau$ field along the $x$ axis is heterogeneous. The magnitude of decrease of $P_{\tau}^{2}\left(L_{x}, L_{y}\right)$ with increasing $L_{x}$ and $L_{y}$ depends 

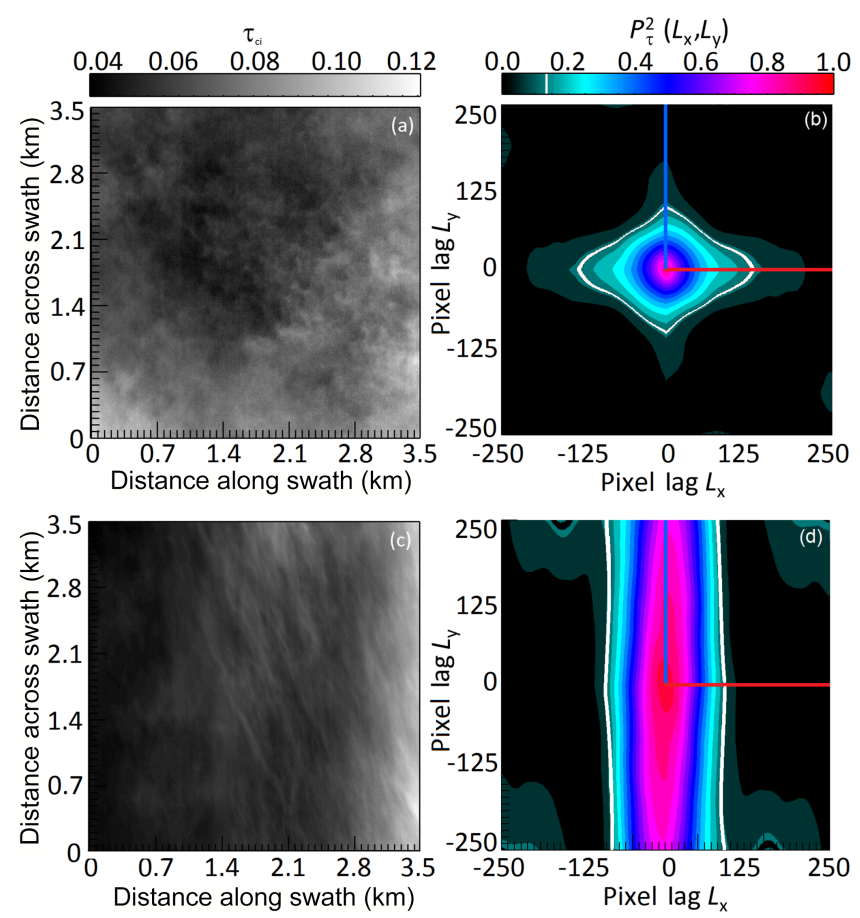

Figure 2. (a) Selected cloud scene $(3.5 \mathrm{~km}$ by $3.5 \mathrm{~km})$ for field of $\tau_{\text {ci }}$ from case Ci-01. (b) Colour-coded 2-D field of $P_{\tau}^{2}\left(L_{x}, L_{y}\right)$, calculated for field of $\tau_{\mathrm{ci}}$ from panel (a). The blue and red lines illustrate the pixel lags selected for the illustration in Fig. 3a. The white line illustrates $\xi_{\tau}$ at $P_{\tau}^{2}\left(L_{x}, L_{y}\right)=1 / \mathrm{e}^{2}$. (c) Same as panel (a) for case Ci-03. (d) Same as panel (b) for selected $\tau_{\text {ci }}$ field shown in panel (c).

on the horizontal structure of the cloud inhomogeneities. The $P_{\tau}^{2}\left(L_{x}, L_{y}\right)$ calculated from Ci-01 (Fig. 2b) show a decrease independent of the direction. In contrast, the $P_{\tau}^{2}\left(L_{x}, L_{y}\right)$ calculated from Ci-03 (Fig. 2d) show a directional dependence.

The squared spatial autocorrelation functions $P_{\tau}^{2}\left(L_{x}, L_{y}\right)$ are used to calculate the decorrelation length $\xi_{\tau}=\sqrt{L_{x}^{2}+L_{y}^{2}}$ implicitly defined by

$P_{\tau}^{2}\left(\xi_{\tau}\right)=\frac{1}{e^{2}}$

Here, $\xi_{\tau}$ quantifies the length scale (in metres) where individual cloud parcels are decorrelated; it provides a measure of the horizontal extent of cloud inhomogeneities. Strong inhomogeneities correspond to small $\xi_{\tau}$. In Fig. $2 \mathrm{~b}$ and d, $\xi_{\tau}$ is indicated by a white line. For $\mathrm{Ci}-01, \xi_{\tau}$ forms a circular shape, indicating a similar magnitude of cloud inhomogeneities in all directions of the cloud field. Conversely, for $\mathrm{Ci}-03, \xi_{\tau}$ along pixel lag $L_{x}$ is significantly smaller than $\xi_{\tau}$ along pixel lag $L_{y}$. This directional dependence is related to the structure of the cloud with regular filaments in the swath direction of the image in Fig. 2c. For case Ci-01, the symmetry in $P_{\tau}^{2}\left(L_{x}, L_{y}\right)$ means that the cloud inhomogeneity can be characterized by a single value $\xi_{\tau}$, independent of direction. For regularly structured clouds such as $\mathrm{Ci}-03$, however,

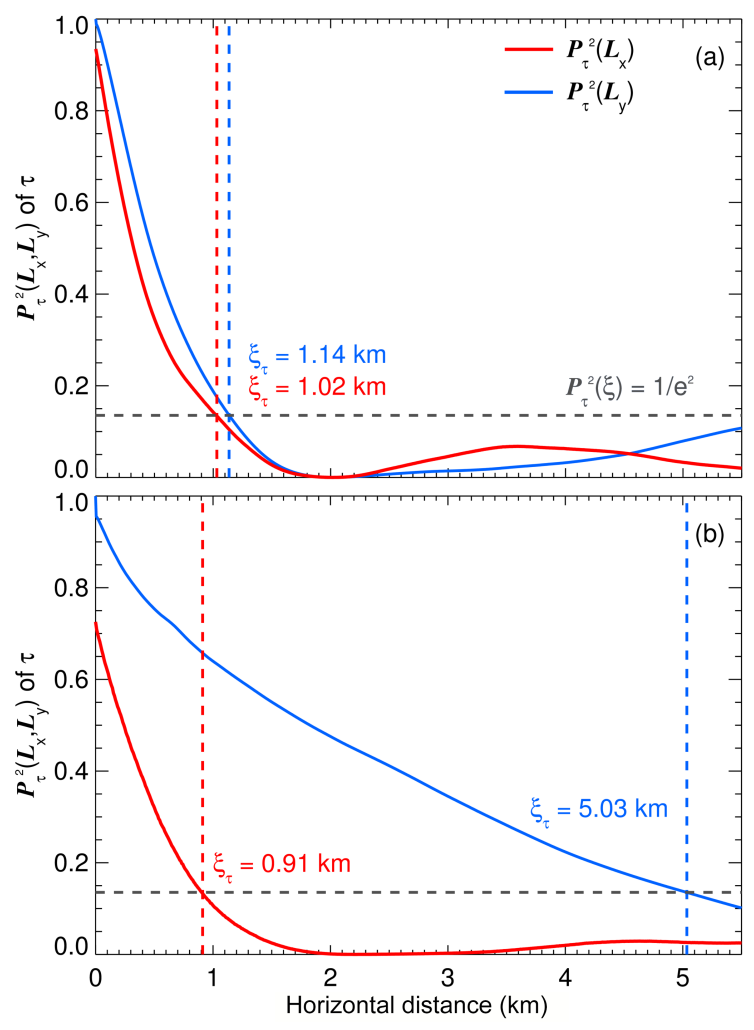

Figure 3. (a) Average of squared 1-D autocorrelation functions $P_{\tau}^{2}\left(L_{x}, L_{y}\right)$ (solid lines) calculated for pixels, which are orientated into the direction of the blue $\left(L_{x}\right)$ and red lines $\left(L_{y}\right)$ illustrated in Fig. 2b. The dashed lines mark the derived distance of the decorrelation length $\xi_{\tau}$, where $P_{\tau}^{2}\left(L_{x}\right)$ and $P_{\tau}^{2}\left(L_{y}\right)$ are decreased to $1 / e^{2}$. (b) Same as panel (a) for $P_{\tau}^{2}\left(L_{x}, L_{y}\right)$ shown in Fig. 2 d.

the 2-D decorrelation can be split into a component of the largest variability and another one along the smallest variability of $\tau$. In the cloud fields presented here, both major axes align with the $x$ and $y$ directions. One-dimensional autocorrelation functions along the axes of strongest $(\leftrightarrow$, red line in Fig. $2 b$ and d) and weakest ( $\uparrow$, blue line in Fig. $2 b$ and d) variability are provided in Fig. $3 a$ and $b$. To derive quantitative values for $\xi_{\tau}^{\leftrightarrow}$ and $\xi_{\tau}^{\uparrow}$ in SI units of metre, the pixel lag is transformed into horizontal distances by multiplying the number of pixels by their pixel size.

For case Ci-01 (Figs. 2a, b, and 3a), the derived $P_{\tau}^{2}\left(L_{x}, L_{y}\right)$ along and across the prevailing directional structure are similar and $\bar{\xi}_{\tau}^{\uparrow}=1.14 \mathrm{~km}$ compares well with $\bar{\xi}_{\tau}^{\leftrightarrow}=$ $1.02 \mathrm{~km}$ within the range of standard deviation given in Table 2. For case Ci-03 (Figs. 2c, d, and 3b), the $P_{\tau}^{2}\left(L_{x}, L_{y}\right)$ along and across the prevailing directional structure differ significantly from each other and $\bar{\xi}_{\tau}^{\uparrow}=5.03 \mathrm{~km}$ is about 6 times larger than $\bar{\xi}_{\tau}^{\leftrightarrow}=0.91 \mathrm{~km}$. Thus, for clouds with a prevailing directional structure it is advisable to give variable $\xi_{\tau}$ as a function of observational direction, e.g., by two 
Table 2. Decorrelation length calculated for the retrieved fields of $\tau$ from the CARRIBA (Ci-01-Ci-04) and VERDI (St-01-St-10) campaigns. Vertical arrows $(\uparrow)$ indicate the calculation of $P_{\tau}^{2}\left(L_{x}, L_{y}\right)$ and subsequent derivation of $\xi_{\tau}$ along $L_{y}$, horizontal arrows $(\leftrightarrow)$ along $L_{x}$. Furthermore, $\bar{\xi}_{\tau}$ is the average of all pixels, $\bar{\xi}_{\tau}-\sigma_{\xi}$ is the average minus standard deviation, and $\bar{\xi}_{\tau}+\sigma_{\xi}$ is the average plus standard deviation.

\begin{tabular}{lccc|rrr}
\hline Case & $\begin{array}{r}\bar{\xi}_{\tau}^{\uparrow}-\sigma_{\xi} \\
(\mathrm{km})\end{array}$ & $\begin{array}{r}\bar{\xi}_{\tau}^{\uparrow} \\
(\mathrm{km})\end{array}$ & $\begin{array}{r}\bar{\xi}_{\tau}^{\uparrow}+\sigma_{\xi} \\
(\mathrm{km})\end{array}$ & $\begin{array}{r}\bar{\xi}_{\tau}^{\leftrightarrow}-\sigma_{\xi} \\
(\mathrm{km})\end{array}$ & $\begin{array}{r}\bar{\xi}_{\tau}^{\leftrightarrow} \\
(\mathrm{km})\end{array}$ & $\begin{array}{c}\bar{\xi}_{\tau}^{\leftrightarrow}+\sigma_{\xi} \\
(\mathrm{km})\end{array}$ \\
\hline Ci-01 & 0.72 & 1.14 & 1.62 & 0.62 & 1.02 & 1.48 \\
Ci-02 & 2.16 & 2.47 & 3.42 & 1.12 & 1.24 & 1.39 \\
Ci-03 & 4.42 & 5.03 & 6.34 & 0.62 & 0.91 & 1.41 \\
Ci-04 & 1.20 & 1.58 & 2.12 & 0.71 & 0.82 & 1.26 \\
\hline St-01 & 0.46 & 0.68 & 0.89 & 0.07 & 0.11 & 0.17 \\
St-02 & 0.67 & 1.12 & 1.56 & 0.11 & 0.18 & 0.26 \\
St-03 & 0.37 & 0.43 & 0.55 & 0.11 & 0.17 & 0.26 \\
St-04 & 0.11 & 0.15 & 0.25 & 0.08 & 0.12 & 0.19 \\
St-05 & 0.13 & 0.17 & 0.26 & 0.07 & 0.10 & 0.17 \\
St-06 & 0.23 & 0.30 & 0.37 & 0.06 & 0.09 & 0.15 \\
St-07 & 0.44 & 0.88 & 1.64 & 0.09 & 0.15 & 0.26 \\
St-08 & 0.30 & 0.73 & 1.57 & 0.13 & 0.16 & 0.24 \\
St-09 & 0.11 & 0.14 & 0.24 & 0.05 & 0.09 & 0.16 \\
St-10 & 0.12 & 0.15 & 0.26 & 0.08 & 0.12 & 0.21 \\
\hline
\end{tabular}

parameters, $\xi_{\tau}^{\uparrow}$ along and $\xi_{\tau}^{\leftrightarrow}$ across the prevailing cloud structure.

The decorrelation lengths are calculated for each measurement case along $\left(\bar{\xi}_{\tau}^{\uparrow}\right)$ and across $\left(\bar{\xi}_{\tau}^{\leftrightarrow}\right)$ the prevailing directional structure of the cloud inhomogeneities, which is identified by the 2-D autocorrelation analysis. Table 2 summarizes and Fig. 4 illustrates the resulting $\bar{\xi}_{\tau}^{\uparrow}$ (blue bars) and $\bar{\xi}_{\tau}^{\leftrightarrow}$ (red bars). Additionally, $\bar{\xi}_{\tau} \pm \sigma_{\xi}$ are included $\left(\bar{\xi}_{\tau}^{\uparrow} \pm \sigma_{\xi}, \bar{\xi}_{\tau}^{\leftrightarrow} \pm \sigma_{\xi}\right)$ in Table 2. Those values illustrate the pixel-by-pixel variability for the calculated $P_{\tau}^{2}\left(L_{x}, L_{y}\right)$ along one direction. Due to the exponential behaviour of $P_{\tau}^{2}\left(L_{x}, L_{y}\right)$, they are asymmetric with respect to $\bar{\xi}_{\tau}^{\uparrow}$ and $\bar{\xi}_{\tau}^{\leftrightarrow}$.

The results show that the observed subtropical cirrus cases yield larger decorrelation lengths $\bar{\xi}_{\tau}$ than the Arctic stratus cases. Thus, the subtropical cirrus cases are more homogeneous than the Arctic stratus cases. Furthermore, the results indicate that for most of the measurement cases a distinct directional structure of cloud inhomogeneities is observed. The results for $\bar{\xi}_{\tau}^{\uparrow}$ are more than twice as large as for $\bar{\xi}_{\tau}^{\leftrightarrow}$ in 9 of the 14 investigated cases.

For the subtropical cirrus cases, $\xi_{\tau}$ varies from 0.82 to $5.03 \mathrm{~km}$, depending on the cloud structure and inhomogeneity. The rather inhomogeneous cases $\mathrm{Ci}-01$ and $\mathrm{Ci}-04$, with highly variable $\tau_{\text {ci }}$ on small scales, yield rapidly decreasing $P_{\tau}^{2}\left(L_{x}, L_{y}\right)$ with low $\bar{\xi}_{\tau}$. In contrast, the quite homogeneous cases $\mathrm{Ci}-02$ and $\mathrm{Ci}-03$ yield slowly decreasing $P_{\tau}^{2}\left(L_{x}, L_{y}\right)$ and larger $\overline{\xi_{\tau}}$. The differences between $\bar{\xi}_{\tau}^{\uparrow}$ and $\bar{\xi}_{\tau} \leftrightarrow$ reach up to $82 \%$.

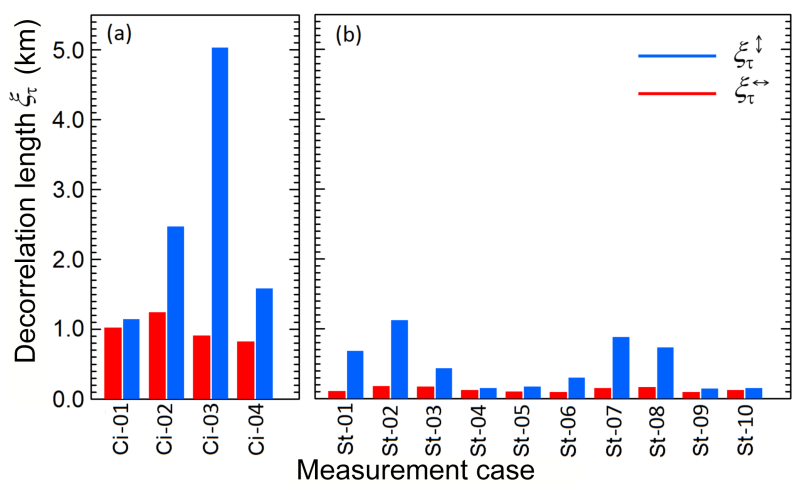

Figure 4. Decorrelation length calculated for the retrieved fields of $\tau$ from the (a) CARRIBA (Ci-01-Ci-04) and (b) VERDI (St-01St-10) campaigns. Vertical arrows $(\uparrow)$ indicate the calculation of $P_{\tau}^{2}\left(L_{x}, L_{y}\right)$ and subsequent derivation of $\xi_{\tau}$ along $L_{y}$, horizontal arrows $(\leftrightarrow)$ along $L_{x}$.

For the Arctic stratus fields observed during VERDI, $\bar{\xi}_{\tau}^{\uparrow}$ and $\bar{\xi}_{\tau}^{\leftrightarrow}$ range between 0.09 and $1.12 \mathrm{~km}$. Similar to the CARRIBA cirrus cases, the differences between $\bar{\xi}_{\tau}^{\uparrow}$ and $\bar{\xi}_{\tau}^{\leftrightarrow}$ are significant, reaching values of up to $84 \%$.

However, the absolute values of $\bar{\xi}_{\tau}^{\hat{\downarrow}}$ and $\bar{\xi}_{\tau}^{\leftrightarrow}$ for the Arctic stratus cases are smaller (more inhomogeneous) than those for the subtropical cirrus cases, although the 1-D inhomogeneity parameters from Table 1 yield similar values. This reveals that the 1-D inhomogeneity parameters $\rho_{\tau}, S_{\tau}$, and $\chi_{\tau}$ just provide incomplete information for a comparison of different types of clouds since they are not able to consider the horizontal structure of cloud inhomogeneities. Differences can only be observed by an evaluation of the horizontal pattern of the cloud inhomogeneities.

\section{Power spectral density analysis}

Multiple scattering in inhomogeneous 1-D cloud structures causes a smoothing of the reflected radiances $I_{\lambda}$ above clouds (Cahalan and Snider, 1989; Marshak et al., 1995). This effect generates uncertainties in the retrieved fields of $\tau$ if homogeneous plane-parallel clouds are assumed in the retrieval. Therefore, in this paper the smoothing effect is analysed using the Fourier transform of the retrieved fields of $\tau$. The application of Fourier transforms for the investigation of cloud inhomogeneities is widely used in the existing literature (e.g., Cahalan, 1994; Davis et al., 1999a; Schröder, 2004). However, in most of these studies, the 1-D Fourier transformation is adopted to narrow pixel lines of radiative quantities such as $I_{\lambda}$ or the reflectivity $\gamma_{\lambda}$. Here, a 2-D Fourier transformation is applied to spatial 2-D cloud scenes. Schäfer et al. (2013) showed that angular features of the scattering phase functions are imprinted in the $I_{\lambda}$ measurements of AisaEAGLE. To avoid artefacts in the Fourier transform arising from those features, fields of $\tau$ are used for the analysis. 

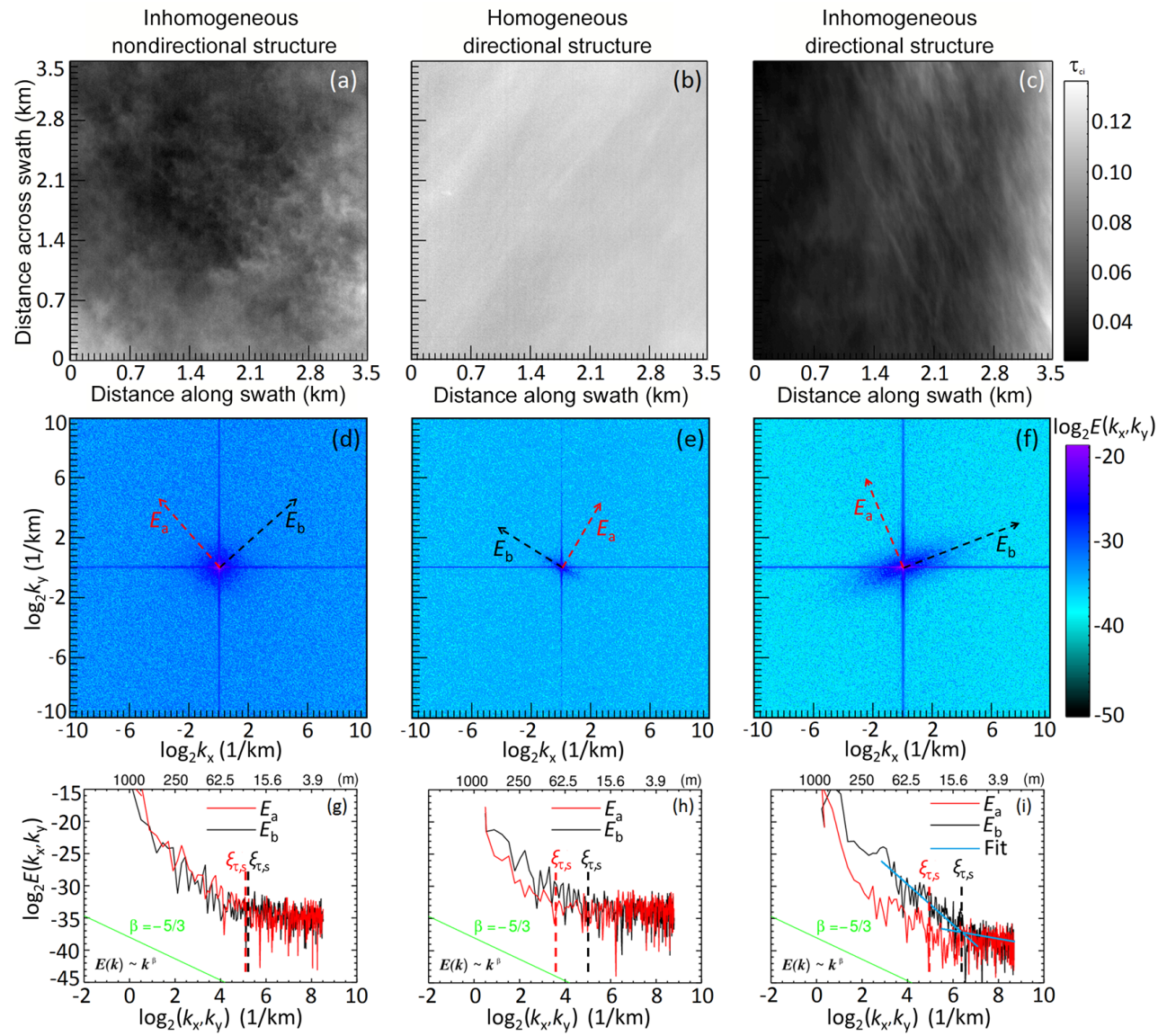

Figure 5. (a-c) AisaEAGLE image $(3.5 \mathrm{~km}$ by $3.5 \mathrm{~km})$, (d-f) 2-D power spectral density $E\left(k_{x}, k_{y}\right)$, and (g-i) 1 -D power spectral density $E\left(k_{x}, k_{y}\right)$ across (red arrows, $\left.E_{a}\right)$ and along (black arrows, $\left.E_{b}\right)$ the prevailing direction of scale-invariant areas for (a, d, g) inhomogeneous cloud without directional structure, $(\mathbf{b}, \mathbf{e}, \mathbf{h})$ homogeneous cloud with slight directional structure, and (c, f, i) inhomogeneous cloud with distinct directional structure. The $\xi_{\tau, \mathrm{s}}$ are marked by dashed coloured lines.

The Fourier transformation decomposes a periodic function into a sum of sinusoidal base functions. For a given measurement, here $\tau(x, y)$, the 2-D Fourier transform $\mathcal{F}_{\tau}\left(k_{x}, k_{y}\right)$ is defined by

$\mathcal{F}_{\tau}\left(k_{x}, k_{y}\right)=\int_{-\infty}^{\infty} \int_{-\infty}^{\infty} \tau(x, y) \times e^{-2 \pi i \times\left(k_{x} x+k_{y} y\right)} \mathrm{d} x \mathrm{~d} y$.

The base functions are described by a complex exponential of different frequency. The fields of $\tau$ are given as a function of horizontal distances $x$ and $y$. Therefore, wave numbers $k_{x}=1 / x$ and $k_{y}=1 / y$ are used in the base functions.

The Fourier coefficients $\mathcal{F}_{\tau}\left(k_{x}, k_{y}\right)$ are calculated using a discrete Fourier transform (DFT). With $n$ and $m$ discrete elements in the $x_{j}$ and $y_{k}$ dimension of the $\tau$ field, the 2-D DFT is derived by

$$
\begin{aligned}
\operatorname{DFT}\left(k_{x}, k_{y}\right)= & \frac{1}{n \times m} \sum_{x_{j}=0}^{n-1} \sum_{y_{k}=0}^{m-1} \tau\left(x_{j}, y_{k}\right) \\
& \times e^{-2 \pi i \times\left(\frac{k_{x} x_{j}}{n}+\frac{k_{y} y_{k}}{m}\right)} .
\end{aligned}
$$

Figures 5 and 6 present the Fourier transform in the form of power spectral densities $E\left(k_{x}\right)$ and $E\left(k_{y}\right)$, in the following called $E\left(k_{x}, k_{y}\right)$, calculated from the complex Fourier coefficients by

$E\left(k_{x}, k_{y}\right)=\operatorname{DFT}^{2}\left(k_{x}, k_{y}\right)$.

Figure 5a-c show $\tau_{\mathrm{ci}}$ fields of three selected cloud areas of $3.5 \mathrm{~km}$ by $3.5 \mathrm{~km}$ size extracted from the cases Ci-01, $\mathrm{Ci}-02$, and $\mathrm{Ci}-03$. Ci-01 represents an inhomogeneous subtropical cirrus case without a preferred direction in the cloud structure (Fig. 5a). In Ci-02 a homogeneous subtropical cirrus case with a moderate directional structure (Fig. 5b) is 

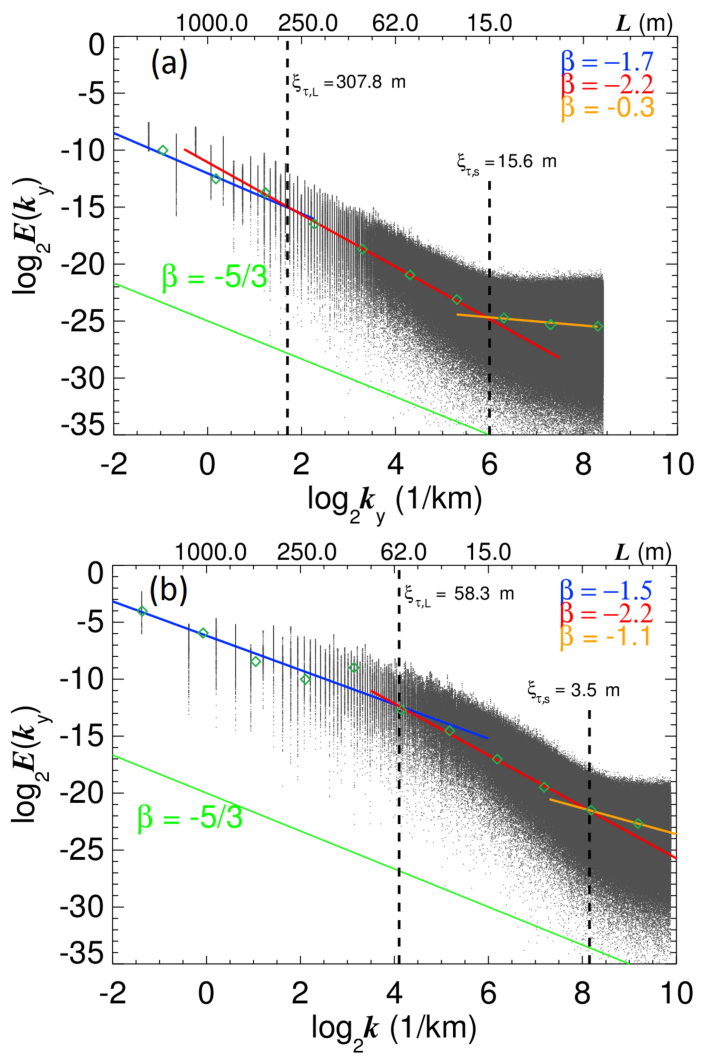

Figure 6. One-dimensional power spectral density $E\left(k_{y}\right)$ (gray dots) for each spatial pixel on the swath axis of the $\tau$ field from (a) case $\mathrm{Ci}-01$ and (b) case St-07. Scale-invariant slopes $\beta$ are marked with solid coloured lines. The $E\left(k_{\mathrm{rn}}\right)$ derived from the octave binning are included as dark green diamonds. Scale breaks $\xi_{\tau, \mathrm{L}}$ and $\xi_{\tau, \mathrm{s}}$ are indicated by dashed lines.

selected, while in $\mathrm{Ci}-03$ an inhomogeneous subtropical cirrus case with a distinct directional structure (Fig. 5c) is presented. Figure $5 \mathrm{~d}-\mathrm{f}$ show the corresponding logarithm of the 2-D power spectral densities $E\left(k_{x}, k_{y}\right)$. The largest values of $E\left(k_{x}, k_{y}\right)$ are found at the smallest wave numbers $k_{x}$ and $k_{y}$, which are located in the centre of the image. In general the values of $E\left(k_{x}, k_{y}\right)$ decrease with increasing $k_{x}$ and $k_{y}$. Inhomogeneous clouds (Fig. 5d and f) show higher values of $E\left(k_{x}, k_{y}\right)$ over a wide range of wave numbers $k_{x}$ and $k_{y}$, whereas the dominating $E\left(k_{x}, k_{y}\right)$ for homogeneous clouds (Fig. 5e) are only located close to the smallest wave numbers $k_{x}$ and $k_{y}$. Similar to the autocorrelation functions, the decrease of $E\left(k_{x}, k_{y}\right)$ is rotationally symmetric for clouds with no preferred directional structure (Fig. 5d), but asymmetrical for clouds with a prevailing directional structure (Fig. 5e, f).

To quantify the 2-D nature of the symmetry, Fig. $5 \mathrm{~g}-$ f show the $E\left(k_{x}, k_{y}\right)$ along (black, $\left.E_{b}\right)$ and across (red, $E_{a}$ ) the direction of the strongest symmetry axis. For the inhomogeneous case without a prevailing directional structure (Ci01 ), both components $E_{a}$ and $E_{b}$ are almost identical. For the homogeneous case with a moderate directional structure
(Ci-02), both $E_{a}$ and $E_{b}$ are similar over most of the covered range of $k_{x}$ and $k_{y}$, except for the smallest wave numbers $k_{x}<3 \mathrm{~km}^{-1}$ and $k_{y}<3 \mathrm{~km}^{-1}$. For the inhomogeneous case with a distinct directional structure (Ci-03), both $E_{a}$ and $E_{b}$ are of similar magnitude only at $k_{x}>7 \mathrm{~km}^{-1}$ and $k_{y}>7 \mathrm{~km}^{-1}$. The differences in $E_{a}$ and $E_{b}$ of clouds with a prevailing directional structure result from the different $k_{x}$ and $k_{y}$, at which the signal turns into white noise (constant $E\left(k_{x}, k_{y}\right)$, independent of $k_{x}$ and $\left.k_{y}\right)$.

This transition is used to characterize the small-scale break $\xi_{\tau, \mathrm{s}}$, which determines the lower size range of the detected cloud inhomogeneities and identifies the scale at which the measurements turn into white noise. To derive $\xi_{\tau, \mathrm{s}}$, fits are applied to the two scale-invariant regimes of $E\left(k_{x}, k_{y}\right)$ (shown for $E_{b}$ in Fig. 5i). Subsequently, the small-scale break $\xi_{\tau, \mathrm{s}}$ is determined as the intersection of those fits. The small-scale break $\xi_{\tau, \mathrm{s}}$ is connected to the pixel size, which depends on the distance between cloud and sensor. The corresponding $k_{x}$ and $k_{y}$ give $\xi_{\tau, \mathrm{s}}$. The small-scale breaks $\xi_{\tau, \mathrm{s}}\left(E_{a}\right)$ and $\xi_{\tau, \mathrm{s}}\left(E_{b}\right)$ for case Ci-01 are at about $0.03 \mathrm{~km}\left(\log _{2} k_{x, y} \approx\right.$ 5). For Case Ci-02, $\xi_{\tau, \mathrm{s}}\left(E_{a}\right)$ and $\xi_{\tau, \mathrm{s}}\left(E_{b}\right)$ are in the length range of $0.09 \mathrm{~km}\left(\log _{2} k_{x, y} \approx 3.5\right)$ and $0.03 \mathrm{~km}\left(\log _{2} k_{x, y} \approx\right.$ $5)$, respectively. The small-scale break $\xi_{\tau, \mathrm{s}}\left(E_{a}\right)$ from case $\mathrm{Ci}-03$ is at about $0.03 \mathrm{~km}\left(\log _{2} k_{x, y} \approx 5\right)$, while $\xi_{\tau, \mathrm{s}}\left(E_{b}\right)$ is at about $0.01 \mathrm{~km}\left(\log _{2} k_{x, y} \approx 6.5\right)$, which is already close to the pixel size, which corresponds to the lower detection limit that leads to white noise. Thus, $\xi_{\tau, \mathrm{s}}$ yields quantitatively larger values along the prevailing cloud structure than across. Furthermore, the ranges of the derived small-scale breaks $\xi_{\tau, \mathrm{s}}$ are found to be close to the ranges of the small-scale breaks reported in literature. Davis et al. (1999b) derived small-scale breaks for a broken-stratocumulus-towering cumulus cloud complex from LWC measurements with a particulate volume monitor probe $(4 \mathrm{~cm}$ resolution) at ranges of about $2-5 \mathrm{~m}$. They proposed that those small-scale breaks are related to extreme values in the detected LWC, which appear on small horizontal scales. Aside from Poissonian fluctuations of the cloud optical thickness $\tau$ and the white noise related to power spectral signals at scales below the pixel size, this might be a further explanation for the derived small-scale breaks in the current study and needs to be investigated in further studies.

Marshak et al. (1995) discussed cloud inhomogeneity and horizontal photon transport being scale-dependent processes. The $E\left(k_{x}, k_{y}\right)$ of cloud optical and microphysical properties are proportional to $k_{x}^{\beta}$ and $k_{y}^{\beta}$, where $\beta$ is the slope of the power spectral density. On large scales, the $E\left(k_{x}, k_{y}\right)$ of $I_{\lambda}, \tau$, LWC, or IWC, for example, follow Kolmogorov's $\beta=-5 / 3$ law of energy distribution in a turbulent fluid (Kolmogorov, 1941). On these scales, the variability in the radiation field follows the variability in LWC. Increasing cloud inhomogeneity causes a decrease of $\beta$ of optical properties on smaller scales, but not in $\beta$ of microphysical properties. On scales influenced by horizontal photon transport, $\beta$ may differ from $-5 / 3$ depending on the cloud inhomogeneity that 
changes the magnitude of horizontal photon transport. Typically, this affects horizontal scales smaller than $1000 \mathrm{~m}$. The higher the cloud inhomogeneity, the larger the deviation from $-5 / 3$. Thus, the slope $\beta$ at scales below $1000 \mathrm{~m}$ provides a measure of cloud inhomogeneity. Usually, the scale break $\xi$ is used to quantify the deviation from $-5 / 3$. In the following, the horizontal scale at which the power spectrum starts to deviate from the $-5 / 3$ law defines the large-scale break $\xi_{\tau, \mathrm{L}}$. The position of the large-scale break depends on the size of the horizontal cloud structures; more inhomogeneous clouds with larger variability on smaller scales yield smaller $\xi_{\tau, \mathrm{L}}$. For scales smaller than $\xi_{\tau, \mathrm{L}}$, the radiative smoothing leads to uncertainties in 1-D cloud retrievals, where the horizontal photon transport is automatically neglected (Cahalan, 1994; Marshak et al., 1998; Zinner et al., 2006; Varnai and Marshak, 2007).

A comparison of the $E\left(k_{x}, k_{y}\right)$ to the $-5 / 3$ law in Fig. $5 \mathrm{~g}$ to $h$ shows that the analysed scenes are too small to cover the larger scales, which are necessary to identify $\xi_{\tau, \mathrm{L}}$. The range of $k_{x}$ and $k_{y}$ is lower than $\xi_{\tau, \mathrm{L}}$, and $E\left(k_{x}, k_{y}\right)$ already exhibit a steeper slope than $\beta=-5 / 3$. Therefore, the size of the selected areas was extended. Unfortunately, this is only possible for calculations of the DFT along $L_{y}$ (across swath). Calculations along $L_{x}$ (swath) do not cover a sufficiently large distance to derive quantitative values for $\xi_{\tau, \mathrm{L}}$. Therefore, the following analysis is performed using 1-D DFT along $L_{\mathrm{y}}$ only. Furthermore, both cloud cases, subtropical cirrus and Arctic stratus, exhibit a similar pixel length along $L_{\mathrm{y}}(5 \pm 2 \mathrm{~m})$, which results from the chosen frame rate (subtropical cirrus: $4 \mathrm{~Hz}$, Arctic stratus: $30 \mathrm{~Hz}$ ) and given cloud $\left(\approx 20 \mathrm{~m} \mathrm{~s}^{-1}\right)$ and aircraft $\left(\approx 70 \mathrm{~m} \mathrm{~s}^{-1}\right)$ velocity. This allows a direct comparison between these two different cloud types with different observation geometry.

Figure $6 a$ and $b$ show the 1-D DFT calculated across the swath for two typical cases of subtropical cirrus (CARRIBA case $\mathrm{Ci}-01$ ) and Arctic stratus (VERDI case St-07). The two cases are selected because they exhibit a similar length $L_{y}$. For each line of the $\tau$ field (each swath pixel) $E\left(k_{y}\right)$ is calculated and the individual power spectra are overlaid as grey dots in Fig. 6. To evaluate the resulting 1-D Fourier spectra with reduced noise $(\mathrm{rn})$ characteristics, the $E_{\mathrm{rn}}(k) \sim k^{\beta}$ are calculated with the use of octave binning, following the method proposed by Davis et al. (1996), Harris et al. (1997), and Schröder (2004). Logarithmically spaced bins $k_{n}$ are calculated by

$k_{n}=\frac{1}{2^{n}} \sum_{i=2 n}^{2^{n+1}-1} k_{i}, n=1,2, \ldots, \log _{2}(N-2)$,

for the number of data points $N . E\left(k_{\mathrm{rn}}\right)$ is then obtained by

$$
E\left(k_{\mathrm{rn}}\right)=\frac{1}{2^{n}} \sum_{i=2 n}^{2^{n+1}-1} E\left(k_{i}\right), n=1,2, \ldots, \log _{2}(N-2) \text {. }
$$

Within each bin $2^{n}$ data points are averaged. In addition to the reduced noise of $E\left(k_{\mathrm{rn}}\right)$ compared to $E\left(k_{y}\right)$ the binning provides a uniform contribution of all scales to the average values.

The $E\left(k_{\mathrm{rn}}\right)$ derived from the octave binning are included as green diamonds in Fig. 6 . The data of the octave binning were used to fit the spectra for different slopes in the different scale ranges. A green line indicates the $\beta=-5 / 3$ law. For large scales, the $E_{\mathrm{rn}}\left(k_{y}\right)$ (blue fit) approximately follow the $-5 / 3$ relation in both cases. The large-scale break $\left(\xi_{\tau, \mathrm{L}}\right)$ is evident at the intersection between the blue and the red line. Here, the slope in the $E_{\mathrm{rn}}\left(k_{y}\right)$ becomes steeper. For the CARRIBA case, $\xi_{\tau, \mathrm{L}}=0.31 \mathrm{~km}$ and the middle-scale slope $\beta_{\mathrm{m}}$ decreases to -2.2 . For the VERDI case $\xi_{\tau, \mathrm{L}}=0.06 \mathrm{~km}$ and $\beta_{\mathrm{m}}$ decreases to -2.2 . The middle-scale slope $\beta_{\mathrm{m}}$ is a function of the inhomogeneity in the measured signals. With increasing inhomogeneity of the optical thickness $\tau, \beta_{\mathrm{m}}$ decreases. Together with the smaller $\xi_{\tau, \mathrm{L}}$, this indicates that the selected Arctic stratus case is more inhomogeneous compared to the selected subtropical cirrus case. As discussed above, $\xi_{\tau, \mathrm{s}}$ is observed at the intersection between the fits for the middle (red, $\beta_{\mathrm{m}}$ ) and small scales (orange, $\beta_{\mathrm{s}}$ ). Due to the analysis of a significantly larger distance compared to Fig. 5, it is highly uncertain to give quantitative numbers for $\xi_{\tau, \mathrm{s}}$. Therefore, it is indicated only qualitatively. However, $\xi_{\tau, \mathrm{s}}$ identifies at which scales the measurements turn into noise. The scale depends on the distance between sensor and cloud. For the sensor, noise dominated at scales 2 times the pixel range, which corresponds to about $15 \mathrm{~m}$ for the subtropical cirrus observations $(\approx 12 \mathrm{~km}$ cloud base altitude) and $3.5 \mathrm{~m}$ for the Arctic stratus observations where the aircraft was closer to cloud top $(\approx 2 \mathrm{~km}$ distance).

Figure 7 illustrates $\xi_{\tau, \mathrm{L}}$ for all available cloud cases from CARRIBA and VERDI. Especially the values for the Arctic stratus are in the size range that was also reported by Marshak et al. (1995), who found scale breaks for fractal clouds in the range of $200-500 \mathrm{~m}$. Furthermore, the values compare well with the derived decorrelation length $\xi_{\tau}$ derived in Sect. 4. Although the exact values of $\xi_{\tau, \mathrm{L}}$ are not equal to $\xi_{\tau}$, both are in the same size range for each individual case. Similar to $\xi_{\tau}$ given in Table $2, \xi_{\tau, \mathrm{L}}$ confirms that $\mathrm{Ci}-02$ and $\mathrm{Ci}-03$ are more homogeneous than $\mathrm{Ci}-01$ and $\mathrm{Ci}-04$. Furthermore, the resulting large-scale breaks $\xi_{\tau, \mathrm{L}}$ confirm the results from the derived decorrelation lengths $\xi_{\tau}$ that the subtropical cirrus observed during CARRIBA are more homogeneous (larger $\xi_{\tau}$ and $\xi_{\tau, \mathrm{L}}$ ) than the Arctic stratus from VERDI (smaller $\xi_{\tau}$ and $\xi_{\tau, \mathrm{L}}$ ). This is related to the fact that $\xi_{\tau, \mathrm{L}}$, which is the radiative smoothing scale, is a function of the cloud geometrical thickness and the transport mean free path. For Arctic stratus both parameters are significantly smaller than for subtropical cirrus.

An estimation of the uncertainty in the derived $\xi_{\tau, \mathrm{L}}$ can be obtained from a comparison to investigations performed by Schröder and Bennartz (2003). Amongst others, Schröder and Bennartz (2003) investigated scale breaks as a function of wavelengths and absorption bands. Their results show uncertainties in a range of 3 to $8 \%$. Schröder and Bennartz 


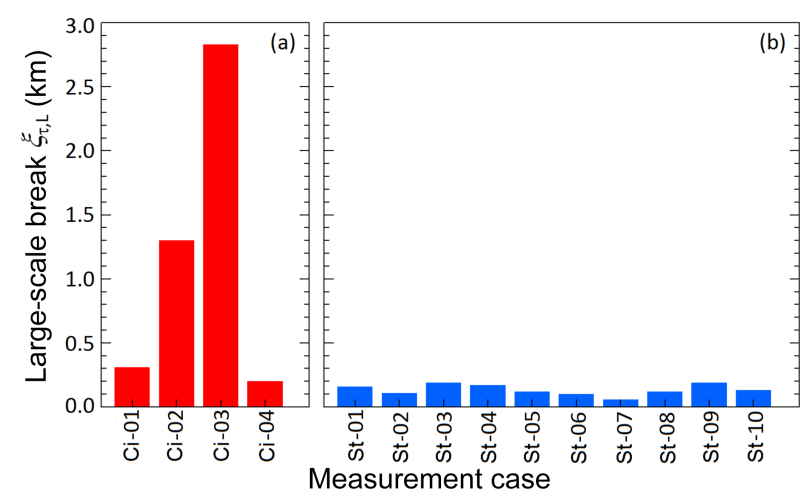

Figure 7. Across swath-derived large-scale breaks, $\xi_{\tau, \mathrm{L}}$ for the retrieved fields of $\tau$ from the (a) CARRIBA (Ci-01 to Ci-04, red) and (b) VERDI (St-01 to St-10, blue) campaigns. The values were derived using the method presented in Fig. 6.

(2003) derived those uncertainty values by subsetting the points of the power spectrum that are used for the slope fit. Using this method, they obtained a set of different slopes and scale breaks. The particular standard deviations of those sets are used as an uncertainty for the octave binning method. Applied to the VERDI cases, the 3 to $8 \%$ result in a maximum uncertainty of $\pm 5 \mathrm{~m}$ (St-07) to $\pm 15 \mathrm{~m}$ (St-03, St-09) in the derived $\xi_{\tau, \mathrm{L}}$. For the CARRIBA cases the maximum uncertainty is in the range of $16(\mathrm{Ci}-04)$ to $226 \mathrm{~m}(\mathrm{Ci}-03)$. However, case $\mathrm{Ci}-03$ is especially characterized as rather homogeneous. Therefore, much lower uncertainty values are to be expected.

\section{Summary and conclusions}

During the two field campaigns CARRIBA and VERDI, downward (ground-based) and upward (measured from aircraft) fields of solar spectral radiance $\left(I_{\lambda}^{\downarrow}, I_{\lambda}^{\uparrow}\right)$ were measured with high spatial resolution (less than $10 \mathrm{~m}$ ), using the imaging spectrometer AisaEAGLE. The measured radiance fields were used to retrieve fields of $\tau$, which were subsequently analysed to quantify horizontal cloud inhomogeneities. Furthermore, due to the observation of 2-D fields, the prevailing directional structure of the cloud inhomogeneities was investigated.

Four subtropical cirrus cases collected during CARRIBA and 10 Arctic stratus cases sampled during VERDI were studied in detail. The cloud inhomogeneity was quantified by three 1-D inhomogeneity parameters $\rho_{\tau}, S_{\tau}$, and $\chi_{\tau}, 1-\mathrm{D}$ and 2-D autocorrelation functions, and Fourier analysis.

Considering the pixel and domain size of the analysed measurements, the results from the calculated 1-D inhomogeneity parameters $\rho_{\tau}$ and $S_{\tau}$ are in agreement with values given in the literature for similar cloud types. The calculated $\rho_{\tau}$ are in the range of $0.17-0.91$ for the subtropical cirrus observed during CARRIBA and $0.15-0.34$ for the Arc- tic stratus measured during VERDI. The literature values are in the range of $0.07-0.78$. The inhomogeneity parameter $S_{\tau}$ exhibits values of 0.08 to 0.48 for CARRIBA and 0.07 to 0.20 for VERDI, which agrees with values of 0.03 to 0.3 given in literature. For $\chi_{\tau}$, the literature estimates values between $\approx 0.65$ and 0.8 , while the results from CARRIBA and VERDI are significantly larger. This is probably related to the different pixel and domain sizes. All values except for $\mathrm{Ci}-04$ $\left(\chi_{\tau}=0.63\right)$ are in the range between 0.92 and 0.99. A further comparison between the results for the clouds encountered during CARRIBA and VERDI showed that all three 1-D inhomogeneity parameters exhibit values of similar magnitude for both cloud types; subtropical cirrus and Arctic stratus. This might lead to the conclusion that the inhomogeneity of both cloud types could be treated by the same 1-D inhomogeneity parameters.

However, the comparison of the 2-D analysis of squared autocorrelation functions $P_{\tau}^{2}\left(L_{x}, L_{y}\right)$ with the 1-D inhomogeneity parameters $\rho_{\tau}, S_{\tau}$, and $\chi_{\tau}$ showed that it is important to consider the full horizontal structure of clouds using 2-D analysis rather than 1-D analysis when determining cloud inhomogeneity. For both cloud cases (subtropical cirrus, Arctic stratus) the 1-D inhomogeneity parameters yield similar values, but significant differences result from the analysis of $P_{\tau}^{2}\left(L_{x}, L_{y}\right)$, which additionally contain information about the horizontal structure of cloud inhomogeneities. The 1-D inhomogeneity parameters are not capable of differentiating the directional structure of clouds and may lead to misinterpretations of cloud inhomogeneity. From the squared autocorrelation functions $P_{\tau}^{2}\left(L_{x}, L_{y}\right)$ the decorrelation length $\xi_{\tau}$ was derived, which is a measure of the size range of the cloud inhomogeneities. The 2-D analysis of $P_{\tau}^{2}\left(L_{x}, L_{y}\right)$ revealed that $\xi_{\tau}$ is a function of the directional structure of the cloud inhomogeneities. Without knowledge of the directional structure of cloud inhomogeneities, no universally valid value for $\xi_{\tau}$ can be derived from the retrieved fields of $\tau$. The differences in $\xi_{\tau}$ as derived from a 1-D autocorrelation analysis along and across the prevailing structure of cloud inhomogeneities reached up to 82 and $84 \%$ for CARRIBA and VERDI, respectively. It is concluded that the directional cloud structure has to be taken into account for a quantification of cloud inhomogeneities. The absolute values of $\xi_{\tau}$ were in the range of 0.82 to $5.03 \mathrm{~km}$ for CARRIBA and 0.09 to $1.12 \mathrm{~km}$ for VERDI. Furthermore, the results from the 2-D analysis showed that for the observed cloud cases the subtropical cirrus was more homogeneous than the Arctic stratus. This result was not available from the investigation of the commonly used 1-D inhomogeneity parameters. Therefore, using 2-D methods in future studies for the characterization of cloud inhomogeneities is advisable since their information content exceeds the information content of the commonly used 2-D inhomogeneity parameters. Today, 2-D images of cloud fields are widespread by measurements of all-sky cameras or satellite observation with high spatial resolution, for example. Applying the presented methods to such continuous 
measurements would provide detailed views into the climatology of cloud inhomogeneities.

Three-dimensional radiative effects are quantified by applying 2-D Fourier transformation to the retrieved fields of $\tau$. The power spectral densities $E\left(k_{x, y}\right)$ calculated from the Fourier transform of $I_{\lambda}^{\downarrow}$ and $I_{\lambda}^{\uparrow}$ show evidence that 3-D radiative effects did affect the radiation field of both cloud types, subtropical cirrus and Arctic stratus. For larger scales (> $1000 \mathrm{~m}$ ), no horizontal photon transport was observed because the $E\left(k_{x, y}\right)$ followed Kolmogorov's $-5 / 3$ law. Approaching smaller scales $(<1000 \mathrm{~m})$, the derived slopes become steeper, indicating radiative smoothing by cloud inhomogeneities and horizontal photon transport. From the intersection of fits of the three slope regimes, the smallscale break $\xi_{\tau, \mathrm{s}}$ (between small- and middle-scale slopes) and the large-scale break $\xi_{\tau, \mathrm{L}}$ (between middle- and large-scale slopes) were derived. Similar to the analysis using autocorrelation functions, $\xi_{\tau, \mathrm{s}}$ depends on the directional structure of the cloud inhomogeneities. Due to a too-small swath width, a similar analysis for $\xi_{\tau, \mathrm{L}}$ could not be performed. However, the calculated $\xi_{\tau, \mathrm{L}}$ along the image are comparable to the results derived from the analysis of $P_{\tau}\left(L_{x}, L_{y}\right)$. The largescale break $\xi_{\tau, \mathrm{L}}$ for CARRIBA was in the range of 0.20 to $2.83 \mathrm{~km}$. For VERDI a range of 0.06 to $0.19 \mathrm{~km}$ was covered by $\xi_{\tau, \mathrm{L}}$.

In early studies, by Marshak et al. (1998) or Schröder (2004) for example, the scale dependence of cloud radiation measurements was analysed along one direction (narrow pixel lines) using 1-D DFT. However, the resulting $E(k)$ are valid for the particular observation direction along the given path only. Due to prevailing wind directions, clouds tend to evolve directional structures. In such cases, the calculated $E(k), \beta, \xi_{\tau, \mathrm{s}}$, and $\xi_{\tau, \mathrm{L}}$ will only be valid for the whole cloud if the cloud structure exhibits a nondirectional character (compare Figs. 2b and 3a). In all other cases, significant differences can be expected (compare Figs. 2d and $3 b)$. We found such differences for more than half of the observed cloud scenes. Therefore, the directional structure of cloud inhomogeneities should be taken into account when cloud inhomogeneities are characterized. It is expected that the information content derived from the directional analysis of cloud inhomogeneities can clearly improve sub-grid scale parametrizations in weather and climate models. For this, depending on the application, the decorrelation length (size and structure of cloud inhomogeneities) or the scale breaks (horizontal photon transport, 3-D radiative effects) may provide better proxies compared to commonly used 1-D inhomogeneity parameters.

However, so far only two cloud types were investigated. To build up a better idea on cloud inhomogeneity of different cloud types, more high definition observations of cloud fields are needed. Aside from dedicated field campaigns, continuous observations by all-sky cameras or satellites with high spatial resolution such as Landsat (15-90 m resolution) or
ASTER (Advanced Spaceborne Thermal Emission and Reflection Radiometer, 15-90 m resolution) may provide the required data.

The 1-D and 2-D autocorrelation functions and Fourier analysis in conjunction with the derived decorrelation length and scale break are helpful tools to verify cloud-resolving models in terms of typical horizontal cloud geometries.

\section{Data availability}

The data used in this study are available upon request from the corresponding author (michael.schaefer@uni-leipzig.de).

Acknowledgements. This study was supported by the German Research Foundation (Deutsche Forschungsgemeinschaft, DFG) as part of the CARRIBA project (WE 1900/18-1 and SI 1534/3-1). We gratefully acknowledge the support by the SFB/TR 172 "ArctiC Amplification: Climate Relevant Atmospheric and SurfaCe Processes, and Feedback Mechanisms (AC) ${ }^{3 \text { " }}$ in Project B03 funded by the DFG. We thank the Max Planck Institute for Meteorology, Hamburg, for supporting the ground-based radiation measurements with the infrastructure of the Barbados Cloud Observatory at Deebles Point on Barbados. We are grateful to the Alfred Wegener Institute Helmholtz Centre for Polar and Marine Research, Bremerhaven, Germany, for supporting the VERDI campaign with the aircraft and manpower. In addition we would like to thank Kenn Borek Air Ltd., Calgary, Canada, for the great pilots who made the complicated measurements possible. For excellent ground support with offices and accommodations during the campaign we are grateful to the Aurora Research Institute, Inuvik, Canada.

Edited by: M. Shupe

Reviewed by: three anonymous referees

\section{References}

Albrecht, B. A.: Aerosols, Cloud Microphysics, and Fractional Cloudiness, Science, 245, 1227-1230, doi:10.1126/science.245.4923.1227, 1989.

Barker, H., Wielicki, B., and Parker, L.: A parameterization for computing grid-averaged solar fluxes for inhomogeneous marine boundary layer clouds. Part II: Validation using satellite data, J. Atmos. Sci., 53, 2304-2316, 1996.

Barlakas, V., Macke, A., and Wendisch, M.: SPARTA - Solver for Polarized Atmospheric Radiative Transfer Applications: Introduction and application in Saharan dust fields, J. Quant. Spectrosc. Ra., 178, 77-92, doi:10.1016/j.jqsrt.2016.02.019, 2016.

Bierwirth, E., Ehrlich, A., Wendisch, M., Gayet, J.-F., Gourbeyre, C., Dupuy, R., Herber, A., Neuber, R., and Lampert, A.: Optical thickness and effective radius of Arctic boundary-layer clouds retrieved from airborne nadir and imaging spectrometry, Atmos. Meas. Tech., 6, 1189-1200, doi:10.5194/amt-6-1189-2013, 2013.

Cahalan, R.: Bounded cascade clouds: albedo and effective thickness, Nonlinear Proc. Geoph., 1, 156-157, 1994. 
Cahalan, R. and Snider, J.: Marine stratocumulus structure, Remote Sens. Environ., 28, 95-107, 1989.

Carlin, B., Fu, Q., Lohmann, U., Mace, G., Sassen, K., and Comstock, J.: High-cloud horizontal inhomogeneity and solar albedo bias, J. Climate, 15, 2321-2339, 2002.

Choi, Y.-S. and Ho, C.-H.: Radiative effect of cirrus with different optical properties over the tropics in MODIS and CERES observations, Geophys. Res. Lett., 33, L21811, doi:10.1029/2006GL027403, 2006.

Davis, A., Marshak, A., Wiscombe, W., and Cahalan, R.: Scale invariance in liquid water distributions in marine stratocumulus. Part I: Spectral properties and stationarity issues, J. Atmos. Sci., 53, 1538-1558, 1996.

Davis, A., Cahalan, R., Spinhirne, D., McGill, M., and Love, S.: Off-beam Lidar: an emerging technique in cloud remote sensing based on radiative Green-function theory in the diffusion domain, Phys. Chem. Earth Pt. B, 24, 177-185, 1999a.

Davis, A., Marshak, A., Gerber, H., and Wiscombe, W.: Horizontal structure of marine boundary layer clouds from centimeter to kilometer scales, J. Geophys. Res., 104, 6123-6144, 1999 b.

Francis, P., Hignett, P., and Macke, A.: The retrieval of cirrus cloud properties from aircraft multi-spectral reflectance measurements during EUCREX '93, Q. J. Roy. Meteor. Soc., 124, 1273-1291, 1998.

Garrett, T. J., Gerber, H., Baumgardner, D. G., Twohy, C. H., and Weinstock, E. M.: Small, highly reflective ice crystals in low-latitude cirrus, Geophys. Res. Lett., 30, 2132, doi:10.1029/2003GL018153, 2003.

Hanus, J., Malenovsky, Z., Homolova, L., Veroslav, K., Petr, L., and Pavel, C.: Potential of the VNIR Airborne hyperspectral system AISA Eagle, in: Symposium GIS, Ostrava, Czech Republic, 2730 January, 2008.

Harris, D., Seed, A., Menabde, M., and Austin, G.: Factors affecting multiscaling analysis of rainfall time series, Nonlinear Proc. Geoph., 4, 137-156, 1997.

Hill, P. G., Hogan, R. J., Manners, J., and Petch, J. C.: Parametrizing the horizontal inhomogeneity of ice water content using CloudSat data products, Q. J. Roy. Meteor. Soc., 138, 1784-1793, 2012.

Huang, D. and Liu, Y.: A novel approach for introducing cloud spatial structure into cloud radiative transfer parameterizations, Environ. Res. Lett., 9, 124022, doi:10.1088/1748-9326/9/12/12402, 2014.

Iwabuchi, H.: Effects of cloud horizontal inhomogeneity on optical remote sensing of cloud parameters, $\mathrm{PhD}$ thesis, Center for Atmospheric and Oceanic Studies, Graduate School of Science, Tokohu University, Sendai, Japan, 2000.

Iwabuchi, H. and Hayasaka, T.: Effects of cloud horizontal inhomogeneity on the optical thickness retrieved from moderateresolution satellite data, J. Atmos. Sci., 59, 2227-2242, doi:10.1175/1520-0469(2002)059<2227:EOCHIO>2.0.CO;2, 2002.

Kolmogorov, A.: Die Energiedissipation für lokalisotrope Turbulenz, Dokl. Akad. Nauk SSSR, 32, 16-18, Nachdruck in: Statistische Theorie der Turbulenz, 1958, edited by: Goering, H., Akademie-Verlag, Berlin, Germany, 71-76, 1941.

Loeb, N. and Davies, R.: Observational evidence of plane parallel model biases: Apparent dependence of cloud optical depth on solar zenith angle, J. Geophys. Res., 101, 1621-1634, doi:10.1029/95JD03298, 1996.

Loeb, N. G., Wielicki, B. A., Doelling, D. R., Smith, G. L., Keyes, D. F., Kato, S., Manalo-Smith, N., and Wong, T.: Toward Optimal Closure of the Earth's Top-of-Atmosphere Radiation Budget, J. Climate, 22, 748-766, doi:10.1175/2008JCLI2637.1, 2009.

Marshak, A., Davis, A., Wiscombe, W., and Cahalan, R.: Radiative smoothing in fractal clouds, J. Geophys. Res., 100, 2624726261, 1995.

Marshak, A., Davis, A., Cahalan, R., and Wiscombe, W.: Nonlocal independent pixel approximation: Direct and inverse problems, IEEE T. Geosci. Remote, 36, 192-204, 1998.

Mayer, B. and Kylling, A.: Technical note: The libRadtran software package for radiative transfer calculations - description and examples of use, Atmos. Chem. Phys., 5, 1855-1877, doi:10.5194/acp-5-1855-2005, 2005.

Oreopoulos, L. and Cahalan, R. F.: Cloud Inhomogeneity from MODIS, J. Climate, 18, 5110-5124, 2005.

Oreopoulos, L., Cahalan, R., Marshak, A., and Wen, G.: A new normalized difference cloud retrieval technique applied to Landsat radiances over the Oklahoma ARM site, J. Appl. Meteorol., 39, 2305-2321, 2000.

Pincus, R., Barker, H. W., and Morcrette, J. J.: A fast, flexible, approximate technique for computing radiative transfer in inhomogeneous cloud fields, J. Geophys. Res., 108, 4376, doi:10.1029/2002JD003322, 2003.

Pinsky, M. and Khain, A.: Fine structure of cloud droplet concentration as seen from Fast-FSSP measurements. Part II: Results of in-situ observations, J. Appl. Meteorol., 42, 65-73, 2003.

Rossow, W. and Schiffer, R.: Advances in understanding clouds from ISCCP, B. Am. Meteorol. Soc., 80, 2261-2287, 1999.

Rozwadowska, A. and Cahalan, R. F.: Plane-parallel biases computed from inhomogeneous Arctic clouds and sea ice, J. Geophys. Res., 107, 4384, doi:10.1029/2002JD002092, 2002.

Schäfer, M., Bierwirth, E., Ehrlich, A., Heyner, F., and Wendisch, M.: Retrieval of cirrus optical thickness and assessment of ice crystal shape from ground-based imaging spectrometry, Atmos. Meas. Tech., 6, 1855-1868, doi:10.5194/amt-6-1855-2013, 2013.

Schäfer, M., Bierwirth, E., Ehrlich, A., Jäkel, E., and Wendisch, M.: Airborne observations and simulations of three-dimensional radiative interactions between Arctic boundary layer clouds and ice floes, Atmos. Chem. Phys., 15, 8147-8163, doi:10.5194/acp15-8147-2015, 2015.

Schröder, M.: Multiple scattering and absorption of solar radiation in the presence of three-dimensional cloud fields, $\mathrm{PhD}$ thesis, Fachbereich Geowissenschaften der Freien Universität Berlin, Berlin, Germany, 2004.

Schröder, M. and Bennartz, R.: Impact of gas absorption and surface albedo on cloud radiative smoothing, Geophys. Res. Lett., 30, 1168-1171, doi:10.1029/2002GL016523, 2003.

Shonk, J. K. P., Hogan, R. J., and Manners, J.: Impact of improved representation of horizontal and vertical cloud structure in a climate model, J. Climate, 38, 2365-2376, doi:10.1007/s00382011-1174-2, 2011.

Siebert, H., Beals, M., Bethke, J., Bierwirth, E., Conrath, T., Dieckmann, K., Ditas, F., Ehrlich, A., Farrell, D., Hartmann, S., Izaguirre, M. A., Katzwinkel, J., Nuijens, L., Roberts, G., Schäfer, M., Shaw, R. A., Schmeissner, T., Serikov, I., Stevens, B., Strat- 
mann, F., Wehner, B., Wendisch, M., Werner, F., and Wex, H.: The fine-scale structure of the trade wind cumuli over Barbados - an introduction to the CARRIBA project, Atmos. Chem. Phys., 13, 10061-10077, doi:10.5194/acp-13-10061-2013, 2013.

Slingo, A.: Sensitivity of the Earth's Radiation Budget to Changes in Low Clouds, Nature, 343, 49-51, 1990.

Stephens, G.: Cloud feedbacks in the climate system: A critical review, J. Climate, 18, 237-273, 2005.

Stocker, T. F., Qin, D., Plattner, G. K., Tignor, M., Allen, S. K., Boschung, J., Nauels, A., Xia, Y., Bex, V., and Midgley, P. M. (Eds.): Climate Change 2013: The Physical Science Basis, Working Group I Contribution to the Fifth Assessment Report of the Intergovernmental Panel on Climate Change, Cambridge University Press, Cambridge, UK, 2013.

Szczap, F., Isaka, H., Saute, M., and Guillemet, B.: Effective radiative properties of bounded cascade nonabsorbing clouds: Definition of the equivalent homogeneous cloud approximation, J. Geophys. Res., 105, 20617-20633, 2000.
Varnai, T. and Marshak, A.: View angle dependence of cloud optical thicknesses retrieved by Moderate Resolution Imaging Spectroradiometer (MODIS), J. Geophys. Res., 112, D06203, doi:10.1029/2005JD006912, 2007.

Wendisch, M., Yang, P., and Ehrlich, A. (Eds.): Amplified climate changes in the Arctic: Role of clouds and atmospheric radiation, vol. 132, chap. 1, 1-34, Sitzungsberichte der Sächsischen Akademie der Wissenschaften zu Leipzig, MathematischNaturwissenschaftliche Klasse, S. Hirzel Verlag, Leipzig, 2013.

Zinner, T., Mannstein, H., and Tafferner, A.: Cb-TRAM: Tracking and monitoring severe convection from onset over rapid development to mature phase using multi-channel Meteosat-8 SEVIRI data, Meteorol. Atmos. Phys., 101, 191-210, 2008

Zuidema, P. and Evans, K.: On the validity of the independent pixel approximation for boundary layer clouds observed during ASTEX, J. Geophys. Res., 103, 6059-6074, 1998. 